\title{
Evidence of subtle departures from Mendelian segregation in a wild lesser kestrel (Falco naumanni) population
}

\author{
JM Aparicio ${ }^{1}, \mathrm{~J} \mathrm{Ortego}^{1,2}, \mathrm{G}_{\text {Calabuig }}{ }^{1}$ and PJ Cordero ${ }^{1}$ \\ ${ }^{1}$ Grupo de Investigación de la Biodiversidad Genética y Cultural, IREC (CSIC-UCLM-JCCM), Ronda de Toledo s/n, Ciudad Real, Spain \\ and ${ }^{2}$ Museo Nacional de Ciencias Naturales (CSIC), José Gutiérrez Abascal 2, Madrid, Spain
}

\begin{abstract}
Some alleles are inherited more frequently than expected from Mendel's rule. This phenomenon, known as transmission ratio distortion (TRD), is found in a broad variety of taxa, but it is thought to be unusual and occurs at a low frequency in any particular population. Here, we used seven microsatellite markers to search for possible TRD in a wild lesser kestrel (Falco naumanni) population. Among the nine alleles analysed with at least 200 known meioses for each sex, we found that two of them (156-AG5 in males and 362-FN1.11 in females) presented subtle $(k=0.6)$ but significant departures from Mendelian segregation. Moreover, in a sample of 53 alleles with at least 15 known meioses, we found a positive correlation between their transmission rates and their frequencies in the population. To
\end{abstract}

estimate the transmission scores for the loci and individuals, we developed a method that allowed us to discover that another locus, FP-46, showed significant TRD, despite the lack of a significant deviation from parity for the alleles considered individually. Finally, we found a consistent transmission bias both within loci and within individuals across loci. Inter-individual differences in TRD support the idea that distorters act over several loci that are evenly distributed across the whole genome, particularly in individuals bearing the distorter alleles. Overall, these findings suggest that TRD might be a more widespread phenomenon than previously revealed by analyses at the allele level.

Heredity (2010) 105, 213-219; doi:10.1038/hdy.2009.173; published online 2 December 2009

Keywords: Falco naumanni; meiotic drive; Mendelian segregation; microsatellite; transmission ratio distortion

\section{Introduction}

Mendel's first law is one of the basic principles of biology and states that each of two alleles from any parent has an equal chance of being transmitted to its progeny. Nevertheless, there are several examples of genes that violate this law, as they are passed from parents to offspring in a proportion higher than the expected 50\% (reviews in Lyttle, 1991, 1993). This phenomenon, known as transmission ratio distortion (TRD)(LeMaire-Adkins and Hunt, 2000), may be the result of different processes: (i) the non-random segregation of chromosomes or alleles during meiosis; (ii) postmeiotic selection because of gamete competition that causes differential viability or fertilization success of haploid gametes; and (iii) postzygotic selection for or against particular genotypes (Pardo-Manuel de Villena et al., 2000; Pardo-Manuel de Villena and Sapienza, 2001).

The two prezygotic processes have attracted the attention of geneticists because they seem to be provoked by segregation distorter genes that subvert meiosis and skew transmission in their own favour, in a phenomenon

Correspondence: Dr JM Aparicio, Grupo de Biodiversidad Genética y Cultural, IREC (CSIC-UCLM-JCCM), Ronda de Toledo s/n, E-13005 Ciudad Real, Spain.

E-mail: josemiguel.aparicio@uclm.es

Received 23 June 2009; revised 30 September 2009; accepted 6 November 2009; published online 2 December 2009 known as meiotic drive (Sandler and Novitski, 1957). In females, the principal opportunity for prezygotic distortion occurs during meiosis, when each primary oocyte produces one functional gamete and three polar bodies. This asymmetry provides an opportunity for 'cheater' genotypes to subvert the segregation process to improve their chances of appearing in the functional gamete (Pardo-Manuel de Villena and Sapienza, 2001). In males, there are some well-documented cases of gamete competition, in which sperm with a particular genotype manage to disrupt or otherwise outperform their competitors, such as the $t$-alleles in the house mouse (Lewontin and Dunn, 1960; Lewontin, 1968; Lyon, 1984) and segregation distorter in Drosophila melanogaster (for example, Hiraizumi et al., 1960; Hartl et al., 1967).

Although segregation distorters are found in a broad variety of taxa, they seem to be unusual and occur at a low frequency in any particular population (Crow, 1991; Hall, 2004). For decades, there has been considerable interest in explaining why these genes are rare given their obvious advantages, as long as they can gain fitness simply by increasing their transmission (for example, Prout et al., 1973; Liberman, 1976; Eshel, 1985; Haig and Grafen, 1991; Weissing and van Boven, 2001; Úbeda and Haig, 2004, 2005). However, although they are difficult to detect, segregation distorters could be more frequent than currently believed (Taylor and Ingvarsson, 2003). In fact, most cases of meiotic drive found so far occur in the best circumstances to be detected, for example when 
there is a strong bias in segregation (normally the transmission rate, $k>0.9$; for example, McMeniman and Barker, 2006); when meiotic drive causes a sex ratio distortion, so that the sex acts as a natural marker (Crow, 1991; Jiggins et al., 1999; Hurst and Werren, 2001; Jaenike, 2008); or when distorters cause deleterious effects on fitness by themselves or by the action of other linked genes (for example, Hiraizumi and Crow, 1960; Buckler et al., 1999). By contrast, in less ideal situations, their detection from the whole genome of an organism can be quite difficult even when distorter genes are common.

Some available evidence indicates that distorters have occurred frequently during the evolutionary history of many species. Some of this evidence comes from experimental crosses between members of closely related species and crosses involving individuals from different isolated populations within a species. Such crosses reveal a high frequency of distorter elements (for example, Dermitzakis et al., 2000; Hall and Willis, 2005; Bratteler et al., 2006; Fishman and Saunders, 2008), which are not detected within the population, probably because most of those distorter genes have already been fixed (Charlesworth and Hartl, 1978). Thus, there is no possibility of unbalanced segregation between either homologous distorter alleles or chromosomes (Hurst and Werren, 2001). If a high number of distorter genes have emerged and been fixed in the evolutionary history of a species, why would these not continue to appear on an ongoing basis today?

We believe that the emergence and existence of distorter genes that have not yet been fixed may also be common in extant populations. However, their effects on TRD could be more easily detected in other alleles than in themselves, especially if these distorters cause segregation bias in other linked elements (alleles or loci) located in the same chromosome, in a process similar to 'hitchhiking' (see Maynard-Smith and Haigh, 1974; Barton, 2000; Chevin and Hospital, 2006). In this case, transmission bias could be frequent among non-distorter loci and/or alleles, although their transmission rate might not be very different from the expected Mendelian segregation ratio. This would occur if the transmission bias of a Responder rapidly decreases with distance to the Distorter, so that the genetic elements with transmission rates subtly different from the parity should be much more frequent than those with high rates of transmission. However, few studies have focused on examining subtle transmission distortions (but see for example Zölner et al., 2004; Bettencourt et al., 2008), as small departures from one-to-one segregation are difficult to detect; moreover, even when found, a natural bias of many investigators is to attribute such departures to viability differences (Hartl, 1975). On the other hand, distorter elements could affect loci rather than particular alleles, as recombination can change the association between the distorter and the different alleles of a locus. Thus, although TRD itself should always occur at the allele level, we could find fair segregation on average for a particular allele in the population and detect biased segregation only at the locus or individual level depending on whether an individual carries the distorter (see also Purushothaman et al., 2008). Measuring the transmission rates of alleles is easy, but measurement at the locus or individual levels is not simple, as the average transmission rate of all alleles of a locus or an individual is invariably $1 / 2$. Therefore, it is necessary to develop a method that allows the study of TRD for loci and individuals.

The aim of this study was to examine the existence of TRD for seven microsatellite markers in a wild lesser kestrel (Falco naumanni) population. First, we assessed any possible transmission bias at the allele level for nine alleles with more than 200 known meioses for each sex. Second, we developed a method that allowed us to score the transmission bias for loci and individuals, to test at the level of loci and individuals for departures from the equal transmission rate, and to measure the repeatability across loci within individuals. Our results support the existence of subtle TRD for two alleles (one for each sex) and two loci and show repeatable TRD among loci within individuals. These results suggest that TRD can be detected at each level of study (that is, alleles, loci and individuals) and may be present at appreciably higher levels (loci and individuals), even when there is an apparent equal segregation of alleles.

\section{Materials and methods}

\section{Organism and field methods}

Since 1991 we have been monitoring a lesser kestrel (F. naumanni) population located in La Mancha, central Spain (Hernández et al., 2007). This species breeds in small colonies of up to 60-70 pairs in our study area. Lesser kestrels lay a single clutch per year, and they can raise up to five young per clutch, although the mean is less than three chicks. The nest sites were located before the onset of laying, and each potential nest was regularly monitored to determine the laying date and clutch size. The eggs were labelled with a waterproof felt-tip pen to identify them individually and determine the fate of each egg. Egg incubation usually lasts 26-27 days. The nests were checked 20 days after the clutch completion to determine possible egg failures during incubation and then a week later to record hatching success. Each chick was marked at hatching with nail varnish or a waterproof felt-tip pen, and they were banded 5-7 days later. The adults were trapped with a noose carpet or by hand during the incubation, measured, and individually marked with metallic and coloured plastic rings (for more details on field procedures, see Aparicio and Cordero, 2001; Aparicio and Bonal, 2002).

Blood samples $(100 \mu \mathrm{l})$ for genetic analyses have been obtained regularly since 2001 by venipuncture of the brachial vein in chicks and adults and preserved in $<1200 \mu \mathrm{l}$ ethanol $96 \%$ at $-20^{\circ} \mathrm{C}$. According to the purpose of this study, we selected families with both parents available for genetic analyses.

\section{Microsatellite genotyping}

We genotyped lesser kestrel families across 11 microsatellite dinucleotide loci, but our analyses for this study were restricted to seven polymorphic microsatellite markers (FP-89, FP-46.1, FP-5, FP-31, Nesje et al., 2000; FN1.11, Ortego et al., 2007; AG5, Topinka and May, 2004; FU-1, JH Wetton, unpublished) that satisfied HardyWeinberg equilibrium to avoid any possible artefacts of PCR. We used QIAamp DNA Blood Mini kits (Qiagen, Hilden, Germany) to extract and purify genomic DNA from the blood samples. Approximately $5 \mathrm{ng}$ of template 
DNA was amplified in $10 \mu \mathrm{l}$ reaction volumes containing $1 X$ reaction buffer $(67 \mathrm{mM}$ Tris-HCL, pH 8.3, $16 \mathrm{mM}$ $\left(\mathrm{NH}_{4}\right)_{2} \mathrm{SO}_{4}, 0.01 \%$ Tween-20, EcoStart Reaction Buffer, Ecogen, Madrid, Spain), $2 \mathrm{mM} \mathrm{MgCl}_{2}, 0.2 \mathrm{mM}$ of each dNTP, $0.15 \mu \mathrm{M}$ of each dye-labelled primer (FAM, HEX or NED) and $0.1 \mathrm{U}$ of Taq DNA EcoStart Polymerase (Ecogen). All reactions were carried out on a Mastercycler EpgradientS (Eppendorf, Hamburg, Germany) thermal cycler. The PCR programme used was a $9 \mathrm{~min}$ denaturation at $95^{\circ} \mathrm{C}$ followed by 30 cycles of $30 \mathrm{~s}$ at $94^{\circ} \mathrm{C}$, $45 \mathrm{~s}$ at the annealing temperature (Ortego et al., 2007) and $45 \mathrm{~s}$ at $72{ }^{\circ} \mathrm{C}$, ending with a $5 \mathrm{~min}$ final elongation stage at $72{ }^{\circ} \mathrm{C}$. The amplification products were electrophoresed using an ABI 310 Genetic Analyzer (Applied Biosystems, Foster City, CA, USA) and the genotypes were scored using GeneMapper 3.7 (Applied Biosystems).

\section{Estimating the transmission scores for loci and individuals $\left(S_{i j}\right)$}

Our purpose was to examine possible TRD at three levels: alleles, loci and individuals. The analysis at the allele level is analytically straightforward whenever a particular allele of the studied microsatellite is closely linked to a hypothetical distorter allele. If so, we would only need to consider the rate of transmission for each allele, which may be defined as the proportion of offspring that inherit the allele from a heterozygous parent. Even when there are differences among individuals in the presence/absence of a distorter element affecting the inherence of an allele, we could detect significant TRD by analysing the simple rate of transmission of an allele in the population. By contrast, if recombination is possible between the distorter allele and the marker examined, we may find cases in which parents do not segregate equally for the alleles of this marker, even when the average transmission rate of each allele within the population is $1 / 2$. Imagine, for example, a locus with two alleles with the same frequency in the population: $D$ (which is a distorter allele) and $d$ (which is normal). This locus is partially linked to a target locus that also has two alleles: $M$ and $m$. In the population, there are $D M / d m$ genotypes, which transmit predominantly the $M$ allele and $d M / D m$ genotypes, which transmit predominantly the $m$ allele. Thus, the transmission rates of both alleles, $M$ and $m$, are equal on average within the population, whereas individuals will show strong deviations from Mendelian expectations depending on their genotypes. In this case, we would need either many offspring of the same parents to analyse the individual transmission rates or a score of the transmission rate that allows a joint analysis of transmission rates of different parents and different alleles of one locus. As we are working in a natural population with a limited number of offspring per parent, we chose the second option. We designed a locus transmission score $(S)$ that allowed us to compare the rates of transmission of different alleles and loci among individuals with different numbers of offspring.

We defined transmission score, $S_{i j}$, for the individual, $i$, and the locus, $j$, as

$$
S_{i j}=\left|\frac{1}{2}-k_{i j g}\right|-\left|\frac{1}{2}-\overline{k_{n}}\right|
$$

The term $\left|1 / 2-k_{i j g}\right|$ is the observed deviation from an equal transmission. Here, $k_{i j g}$ is the transmission rate of one of the two alleles (for example $g$ to designate the greater) of the locus $j$ from a parent $i$ to their $n$ offspring. The second term, $\left|1 / 2-\overline{k_{n}}\right|$, is the expected mean deviation, which is only dependent on the number of offspring examined $(n)$ for any locus or individual.

Note that in the absence of TRD, $S_{i j}$ should be zero for any locus and individual regardless of the number of offspring analysed. Thus, under the null hypotheses of non-biased transmission, we could get $\bar{S}_{i}$ for the $i$ parent by averaging all of the $S_{i j}$ values of all of the loci examined for this individual. Similarly, we can also estimate $\bar{S}_{j}$ for the $j$ locus in a population by averaging all $S_{i j}$ values of all parents examined for this locus.

Prior to the analysis, all data were pre-processed to detect any pedigree errors. Moreover, within each family, we excluded from the analyses any marker locus for which either parent had missing data to avoid possible biases because of misspecified allele frequencies or linkage disequilibrium between the markers.

\section{Results}

We examined the segregation ratios of seven loci that satisfied Hardy-Weinberg equilibrium in 89 heterozygous parents for which we knew their genotype and that of their mates and at least of five of their offspring. The maximum number of offspring typed per parent and locus was 15 and the mean was 7.5 offspring \pm 2.6 (s.d.).

\section{Alleles}

First, we examined the transmission ratios at the allele level. The seven loci analysed in this study totalled to 68 alleles, some of them with low frequencies. To examine the distribution of the segregation ratios reducing statistical variance, we have only considered 53 alleles that were involved in at least 15 meioses. In appearance, segregation ratios were quite close to the expected parity. The highest transmission rate was $0.62(31 / 50)$ for the allele 142 of the locus FP-31, and the lowest was 0.28 (5/18) for the allele 101 of the Locus FP-5. The distribution did not differ from the normal (Kolmogorov-Smirnov: $D=0.12, \quad N=53, P=0.44$; Figure 1). However, the average transmission rate of these 53 alleles differed slightly, but significantly, from 0.5 (mean \pm s.d.: $0.48 \pm 0.07, t=-2.09$, d.f. $=52, P=0.042$ ). This result suggests that transmission rates of some alleles subtly deviated from the parity. Nevertheless, it may be difficult to identify those alleles because a large sample would be necessary to find statistically significant differences when the divergence from Mendelian segregation is small. Hence, to determine what proportion of alleles did not segregate in agreement with Mendel's rule, we restricted our analyses to nine alleles, for which more than 200 meioses were known for each sex. This sample size would only allow us to detect departures from the parity higher than \pm 0.1 , approximately. One of the nine alleles analysed for males (156-AG5) and another for females (362-FN1.11) showed significant departures from equal transmission after applying Bonferroni corrections for multiple tests (Table 1).

Given that the non-Mendelian inheritance of microsatellite alleles can be attributed to artefacts of PCR, such as null alleles and allelic dropout, we examined the transmission rates $(k)$ for sub-samples that included only those cases where both parents did not match any of 


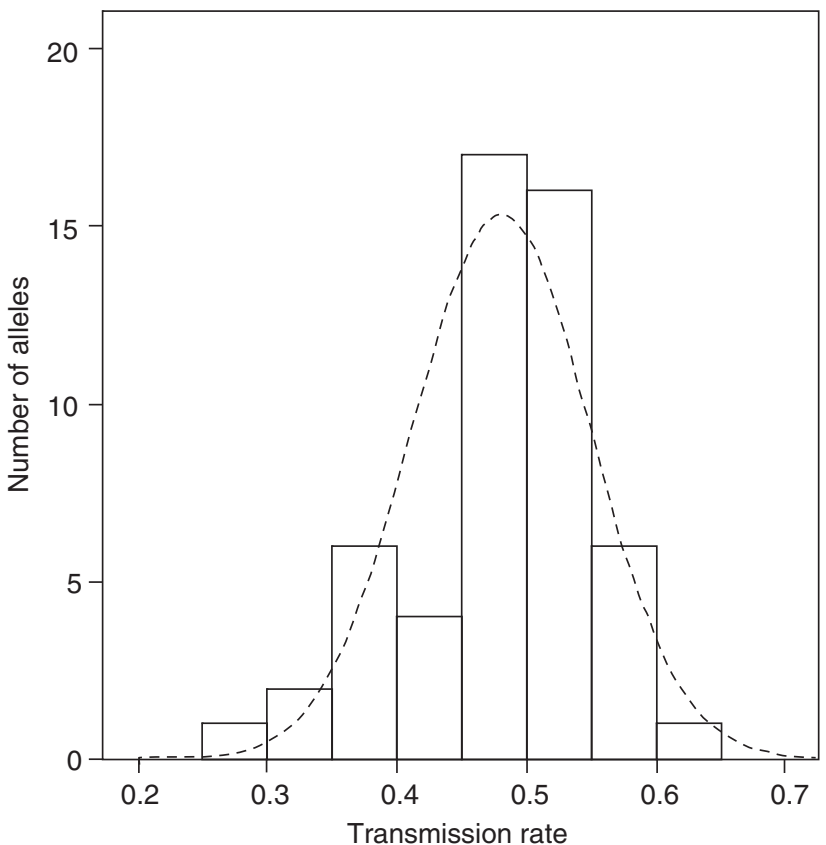

Figure 1 The distribution of transmission rates for 53 alleles with more than 15 known meioses in a wild lesser kestrel population. The dotted line represents the expected normal distribution.

Table 1 The transmission rate $(k)$ for nine alleles with more than 200 known meioses for each sex.

\begin{tabular}{|c|c|c|c|c|c|c|c|c|c|}
\hline \multirow[t]{2}{*}{ Locus } & \multirow[t]{2}{*}{ Allele } & \multicolumn{4}{|c|}{ Females } & \multicolumn{4}{|c|}{ Males } \\
\hline & & $\mathrm{N}$ & $k$ & Z & $\mathrm{P}$ & $\mathrm{N}$ & $k$ & Z & $\mathrm{P}$ \\
\hline P-89 & 119 & 208 & 0.495 & 0.1387 & 0.8898 & 320 & 0.519 & 0.6708 & 0.5028 \\
\hline FP-46.1 & 125 & 319 & 0.542 & 1.5117 & 0.1316 & 309 & 0.547 & 1.6498 & 0.1000 \\
\hline FP-5 & 105 & 314 & 0.525 & 0.9029 & 0.3673 & 283 & 0.527 & 0.8917 & 0.3733 \\
\hline FP-5 & 107 & 221 & 0.466 & 1.0090 & 0.3141 & 295 & 0.492 & 0.2911 & 0.7712 \\
\hline FP-31 & 132 & 349 & 0.499 & 0.0535 & 0.9573 & 347 & 0.496 & 0.1610 & 0.8721 \\
\hline FN1.11 & 362 & 298 & 0.611 & 3.8233 & 0.0002 & 352 & 0.443 & 2.1320 & 0.0337 \\
\hline FN1.11 & 364 & 297 & 0.448 & 1.7988 & 0.0731 & 267 & 0.532 & 1.0404 & 0.2991 \\
\hline AG5 & 156 & 235 & 0.523 & 0.7176 & 0.4737 & 294 & 0.599 & 3.3826 & 0.0008 \\
\hline FU-1 & 120 & 289 & 0.561 & 2.0588 & 0.0404 & 323 & 0.548 & 1.7249 & 0.0855 \\
\hline
\end{tabular}

$Z$ is the standard normal statistic. The bold $P$-values indicate statistical significance after Bonferroni corrections.

their alleles at the examined locus (either AG5 or FN1.11). For 362-FN1.11, the transmission rate did not vary in this sub-sample $(k=0.60, N=57)$ with respect to the rest of the crosses $\left(k=0.61, N=241 ; \chi_{1}^{2}=0.009\right.$, $P=0.93)$, whereas for 156 -AG5, $k$ was even slightly higher in that sub-sample $(k=0.71, N=48)$ than in the other crosses $(k=0.58, N=246)$, although this difference was not statistically significant $\left(\chi_{1}^{2}=2.35, P=0.125\right)$. Therefore, these departures from equal segregation cannot be attributed to artefacts of PCR.

To determine whether these biases in the transmission of alleles were due to a differential viability of zygotes, we analysed the fertility and embryo mortality of eggs produced by kestrel pairs with known genotypes. If there were an effect of the allele on either fertility or viability, we would expect that the homozygous parents for the allele with unequal segregation would have a higher hatching success than heterozygous parents. This would occur because all gametes and zygotes of a homozygous parent would carry that allele, whereas only ca. $1 / 2$ or ca. $3 / 4$ of zygotes produced by the heterozygous parents (depending if only one or both progenitors) would bear that allele. However, neither the proportion of eggs that contain no visible embryo, nor the proportion of embryos that died before hatching, nor the total hatching success differed between the homozygous and heterozygous progenitors for either of these alleles, 156-AG5 (Table 2) and 362-FN1.11 (Table 3).

Although we only found significant deviations from the equal segregation in two alleles, there may have been small deviations that were missed. If such subtle differences are consistent deviations from the parity, one would expect that the alleles with a higher rate of transmission are more frequent in the population. Thus, we examined the relationship between the transmission rate and frequency of the alleles in the population and found a positive and significant correlation between both variables $(r=0.30, N=68 ; P=0.01)$. We repeated the analysis, ruling out the alleles with less than 15 meiotic events to avoid the large statistical noise in their transmission rate estimates, and again we found the same pattern $(r=0.31 ; N=53 ; P=0.02$; Figure 2$)$.

Finally, using an ANOVA with the allele deviance (that is, the absolute difference of the transmission rate relative to $1 / 2$ ) as the dependent variable and the locus identity as the fixed factor, we found that the allele deviances within loci are less variable than between loci $\left(\mathrm{F}_{6,46}=2.96 ; P=0.016\right)$. This suggests that locus-specific factors determine the bias in the transmission rates, which could be simply the distance between the distorter and responder.

\section{Loci and individuals}

For loci analyses at the individual level, we estimated transmission bias scores $\left(S_{i j}\right)$ that control for the number of meiosis to make comparable measures of individuals with different numbers of offspring (see Materials and methods). There was no statistical difference between males and females in $S_{j}$ for any locus, except the FP-31 locus (Mann-Whitney $U$-test: $U=209.5, N_{1}=24, N_{2}=26$, $P=0.044)$, which was also non-significant when Bonferroni corrections for multiple tests were applied (Table 4). Pooling the data from males and females in the analyses, two loci (FP-46.1 and AG5) showed S-values significantly different than the expected from Mendelian segregation after Bonferroni corrections (Table 4).

Furthermore, we analysed whether the biases in the transmission scores were consistent within individuals across different loci. For this analysis, the $S$-values were standardized for each locus to avoid any effects because of differences among loci. Considering individuals with $S$-values for at least five loci, we found that these scores were significantly less variable within individuals than among them $\left(\mathrm{F}_{29,130}=1.56 ; P=0.048\right)$, suggesting the existence of an individual component in the transmission bias.

\section{Discussion}

This study, carried out in a wild lesser kestrel population, showed that the average transmission ratio of 53 alleles belonging to seven loci was subtly but significantly smaller (0.48) than that expected from the Mendelian segregation rule. The fact that these biases were 
Table 2 Egg viability in relation to the heterozygosity of the father for the 156-AG5 allele

\begin{tabular}{|c|c|c|c|c|c|c|c|c|}
\hline & \multicolumn{3}{|c|}{ Homozygous fathers $(156 / 156)$} & \multicolumn{3}{|c|}{ Heterozygous fathers $(156 / x)$} & \multirow[t]{2}{*}{ Wald } & \multirow[t]{2}{*}{$\mathrm{P}$} \\
\hline & Mean & s.d. & $\mathrm{N}$ & Mean & s.d. & $\mathrm{N}$ & & \\
\hline Proportion of eggs with no visible embryo & 0.049 & 0.122 & 75 & 0.047 & 0.121 & 160 & 0.002 & 0.97 \\
\hline Embryo mortality & 0.041 & 0.121 & 74 & 0.041 & 0.129 & 160 & 0.001 & 0.98 \\
\hline Hatching success & 0.886 & 0.172 & 78 & 0.886 & 0.190 & 167 & 0.010 & 0.92 \\
\hline
\end{tabular}

Table 3 Egg viability in relation to the heterozygosity of the mother for the 362-FN1.11 allele

\begin{tabular}{|c|c|c|c|c|c|c|c|c|}
\hline & \multicolumn{3}{|c|}{ Homozygous mothers $(362 / 362)$} & \multicolumn{3}{|c|}{ Heterozygous mothers $(362 / x)$} & \multirow[t]{2}{*}{ Wald } & \multirow[t]{2}{*}{$\mathrm{P}$} \\
\hline & Mean & s.d. & $\mathrm{N}$ & Mean & s.d. & $\mathrm{N}$ & & \\
\hline Proportion of eggs with no visible embryo & 0.034 & 0.087 & 44 & 0.042 & 0.114 & 166 & 0.136 & 0.71 \\
\hline Embryo mortality & 0.038 & 0.123 & 43 & 0.046 & 0.135 & 163 & 0.657 & 0.42 \\
\hline Hatching success & 0.903 & 0.152 & 47 & 0.887 & 0.178 & 172 & 0.808 & 0.37 \\
\hline
\end{tabular}

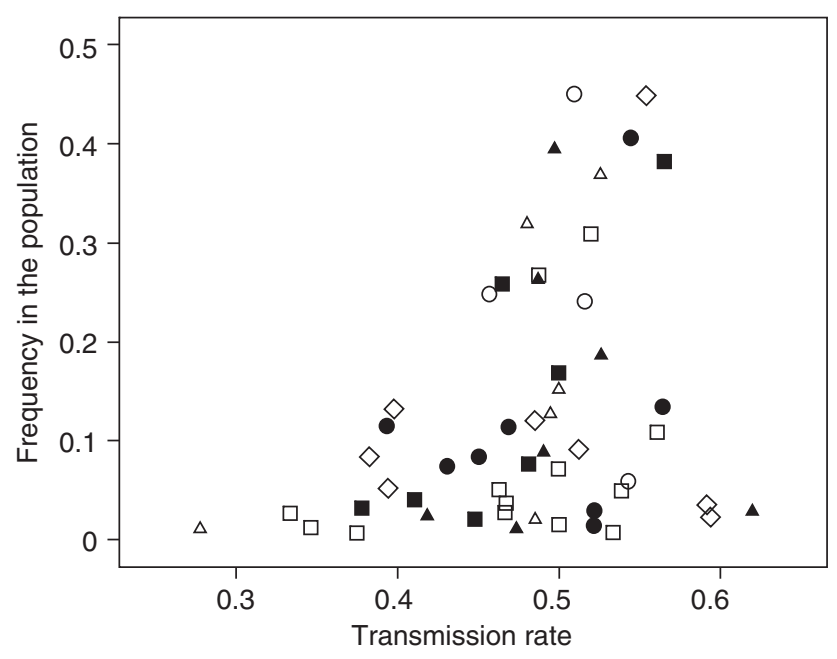

Figure 2 The relationship between the frequencies in the population of 53 alleles and their transmission rates. The alleles of the same locus are represented by equal marks.

moderate makes them difficult to detect, especially in wild populations, as the detection would require an analysis of a large number of meioses. Nevertheless, two alleles, 156-AG5 and 362-FN1.11, of the nine examined with more than 200 known meioses for each sex, presented significant TRD for males and females, respectively. An additional analysis, excluding the data from parents that shared alleles with their mates, also revealed similar departures from Mendelian segregation and, therefore, this analysis rules out that such a TRD was due to artefacts of PCR, such as null alleles and allelic dropout.

Differential embryo viability may also cause TRD. However, this cause was excluded as an explanation of the TDR for both alleles, 156-AG5 and 362-FN1.11. If that were the case, we should have found an embryonic mortality from heterozygous parents at least 10\% higher than that of homozygous parents, but our data showed that there was no difference between them for any allele (Tables 2 and 3). The lack of differences in embryo viability ruled out any postzygotic origin of the observed TRD on those alleles and instead points to prezygotic processes.
In addition, we presented a new method that allows for the examination of possible TRD at the locus and individual levels. Analyses at the locus level revealed that two loci, FP-46 and AG5, showed significant TRD. Although the distortion in AG5 could be explained by the significant bias in the segregation of one of its alleles, no allele considered individually deviated significantly from parity in the case of the FP-46 locus. Several theoretical studies have shown that distorter or modifier loci can differentially alter the segregation of alleles belonging to a single locus (for example, Prout et al., 1973). Therefore, the rate of transmission of a particular allele is greater than that of others at the same locus. However, the results for the FP-46 locus support the idea that the distorter element acts on a region of the chromosome, so that TRD may be detected at the locus and individual levels rather than at the allele level because, among the individuals carrying the supposed distorter element, the target locus may be occupied by an allele in some individuals or by its partner in other ones. Therefore, the equal segregation of alleles does not exclude the existence of TRD processes in their corresponding loci.

Surprisingly, we did not find significant TRD for the locus FN1.11, in spite of the fact that of one of its alleles, 362 , showed a significant bias in the transmission from mothers to offspring. We think that FN1.11 presents a lower level of TRD than other loci such as AG, and the non-significance at the locus level analysis may be due to an insufficient number of mothers sampled for this locus.

This study shows one of the highest proportions in the literature of TRD loci in natural crosses within a population. We believe that TRD could take place in an even higher proportion of loci for two reasons. First, our analyses were restricted to polymorphic microsatellite markers that satisfied the Hardy-Weinberg equilibrium to avoid any possible artefacts of PCR (Callen et al., 1993; McMeniman and Barker, 2006). Monomorphic (or almost monomorphic) markers as well as those that do not comply with HWE are normally excluded from studies with microsatellites because they are inappropriate for analysing parentage or heterozygosity. However, those properties that make them unsuitable for such studies could result from the high bias in their transmission. The sample of microsatellites markers analysed here may 
Table 4 The mean transmission scores $(S)$ for seven loci in parents with at least five genotyped offspring

\begin{tabular}{|c|c|c|c|c|c|c|c|c|c|}
\hline \multirow[t]{2}{*}{ Loci } & \multicolumn{2}{|c|}{ Females } & \multicolumn{2}{|c|}{ Males } & \multicolumn{2}{|c|}{ Comparison $F / M$} & \multicolumn{2}{|c|}{ Both sexes } & \multirow{2}{*}{$\begin{array}{c}\text { Test for non-Mendelian } \\
\text { transmission }^{\mathrm{a}} \\
\mathrm{P}\end{array}$} \\
\hline & $S$ & $\mathrm{~N}$ & $S$ & $\mathrm{~N}$ & $U$ & $\mathrm{P}$ & Mean & $\mathrm{N}$ & \\
\hline FP-89 & -0.0312 & 10 & -0.0188 & 23 & 113 & 0.937 & -0.0226 & 33 & 0.8829 \\
\hline FP-46.1 & 0.0389 & 26 & 0.0416 & 22 & 277 & 0.852 & 0.0402 & 48 & 0.0058 \\
\hline FP-5 & -0.0167 & 26 & -0.0430 & 30 & 326.5 & 0.292 & -0.0308 & 56 & 0.9804 \\
\hline FP-31 & 0.0154 & 24 & -0.0388 & 26 & 209.5 & 0.044 & -0.0128 & 50 & 0.7345 \\
\hline FN1.11 & 0.0141 & 33 & -0.0116 & 35 & 520.5 & 0.482 & 0.0009 & 68 & 0.4855 \\
\hline AG-5 & 0.0652 & 21 & 0.0261 & 22 & 205.5 & 0.534 & 0.0452 & 43 & 0.0063 \\
\hline FU-1 & -0.0097 & 18 & 0.0364 & 24 & 176 & 0.306 & 0.0167 & 42 & 0.1683 \\
\hline
\end{tabular}

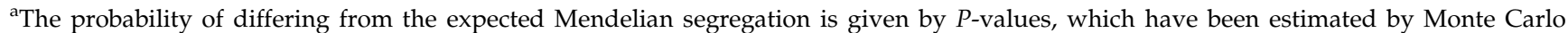
randomisations (10000 iterations) with an equal number of parents and offspring to those available for each sample. The bold values were significant after Bonferroni corrections.

therefore give a conservative estimation of the proportion of loci presenting transmission distortion. Second, there may be subtle deviations from Mendelian segregation for many other alleles that are statistically imperceptible, but consistent. In our sample, there was a close positive correlation between the transmission rates and the frequencies of alleles in the population (Figure 2). Such a correlation cannot be explained by natural selection, because a natural selection process would increment the frequency of the allele but not its transmission rate. It must be considered that artefacts related with PCR could potentially account for such a positive correlation, because the existence of null alleles underestimates both the allele frequency and the transmission ratio. However, it is difficult to imagine that the wide variability in allelic frequencies is caused by null alleles and that PCR artefacts of such magnitude are not detected by a simple test of equilibrium. A more parsimonious explanation may be that the positive correlation between the transmission rates of alleles and their frequencies in the population is due to prezygotic processes that alter segregation.

Mendelian transmission has been considered the rule (for example, Úbeda, 2006). However, there is an increasing agreement, based on studies in a wide variety of organisms, that transmission distortion may be more common than previously thought (Taylor and Ingvarsson, 2003; Purushothaman et al., 2008). For example, studies on plants revealed that nearly half of the markers exhibit significant TRD in intraspecific crosses (for example, Kuittinen et al., 2004, Hall and Willis, 2005, Bratteler et al., 2006, McDaniel et al., 2007). These high levels of distortion might occur because those crosses are generally performed between distant populations, and the level of distortion may depend on the degree of divergence of the genomes (Jenczewski et al., 1997, Hall and Willis, 2005). Nevertheless, these crosses can also reveal the existence of latent distorters that at some time were able to function within the population or could even function in the present, causing only small distortions. In a human population, Zölner et al. (2004) also detected a modest but significant genome-wide shift towards biased genetic similarity between siblings ( $50.43 \%$ for the autosomes), suggesting that the total number of distortion loci in the genome is likely to be large enough to produce a genomewide shift of this magnitude.

At the individual level, we found that genetic transmission biases were significantly less variable within individuals than among them. This result is interesting because it suggests the existence of a mechanism that causes distortion on multiple chromosomes. However, our data are not sufficient to speculate on the existence of one of these mechanisms, because such repeatability across loci could also occur if some markers are located on the same chromosome. Further studies would therefore be necessary to elucidate this issue.

In conclusion, this study reveals transmission bias for two alleles and two loci and shows that TRD are consistent both within loci and across loci within individuals. The fact that transmission biases are likely to decrease and recombination rates increase with the chromosomal distance between the marker and the hypothesized distorter loci may explain why we have observed subtle deviations from Mendelian expectations at the locus level even when there is no global effect on their alleles. Overall, these findings suggest that TRD might be a more widespread phenomenon than revealed earlier by analyses at the allele level and that subtle deviations within loci and individuals should be further considered in the future.

\section{Conflict of interest}

The authors declare no conflict of interest.

\section{Acknowledgements}

The primer sequence for the microsatellite FU-1 was kindly provided by Jon $\mathrm{H}$ Wetton (Forensic Science Service, UK). We also are grateful to Raúl Bonal and three anonymous referees for their valuable comments. We manipulated and banded lesser kestrels under a license from the Spanish institutional authorities (Dirección Gral. de Medio Ambiente Natural, JCCM) and we followed general ethical guidelines for animal welfare and nature conservation. This work has been supported by el Ministerio de Ciencia e Innovación (projects: CGL2005-05611-C02-02/BOS and CGL2008-00095/BOS) and Junta de Comunidades de Castilla-La Mancha (project: PCI08-0130-3954). JO is supported by a postdoctoral JAE-Doc (CSIC) contract and GC by a pre-doctoral fellowship from the Junta de Comunidades de Castilla-La Mancha/European Social Fund (to GC). We performed all of the laboratory work at the Laboratory of Genetics of the IREC and the fragment genotyping was performed by the Centro de Investigaciones Biológicas (CSIC). 


\section{References}

Aparicio JM, Bonal R (2002). Effects of food supplementation and habitat selection on timing of lesser kestrel breeding. Ecology 83: 873-877.

Aparicio JM, Cordero PJ (2001). The effects of the minimum threshold condition for breeding on offspring sex ratio adjustment in the lesser kestrel. Evolution 55: 1188-1197.

Barton NH (2000). Genetic hitchhiking. Philos Trans $R$ Soc London B 355: 1553-1562.

Bettencourt C, Fialho RN, Santos C, Montiel R, Bruges-Armas J, Maciel P et al. (2008). Segregation distortion of wild-type alleles at the Machado-Joseph disease locus, a study in normal families from the Azores islands (Portugal). J Hum Gen 53: 333-339.

Buckler IV ES, Phelps-Durr TL, Buckler CSK, Dawe RK, Doebley JF, Holtsford TP (1999). Meiotic drive of chromosomal knobs reshaped the maize genome. Genetics 153: 415-426.

Bratteler M, Lexer C, Widmer A (2006). A genetic linkage map of Silene vulgaris based on AFLP markers. Genome 49: 320-327.

Callen DF, Thompson AD, Shen Y, Phillips HA, Richards RI, Mulley JC et al. (1993). Incidence and origin of 'null' alleles in the (AC)n microsatellite markers. Am J Hum Genet 52: 922-927.

Charlesworth B, Hartl DL (1978). Population dynamics of the segregation distorter polymorphism of Drosophila melanogaster. Genetics 89: 171-192.

Chevin LM, Hospital F (2006). The hitchhiking effect of an autosomal meiotic drive gene. Genetics 173: 1829-1832.

Crow JF (1991). Why is Mendelian segregation so exact? BioEssays 13: 305-312.

Dermitzakis ET, Masly JP, Waldrip HM, Clark AG (2000). NonMendelian segregation of sex chromosomes in heterospecific Drosophila males. Genetics 154: 687-694.

Eshel I (1985). Evolutionary genetic stability of Mendelian segregation and the role of free recombination in the chromosomal system. Am Nat 125: 412-420.

Fishman L, Saunders A (2008). Centromere-associated female meiotic drive entails male fitness costs in monkeyflowers. Science 232: 1559-1562.

Haig D, Grafen A (1991). Genetic scrambling as a defence against meiotic drive. J Theor Biol 153: 531-558.

Hall DW (2004). Meiotic drive and sex chromosome cycling. Evolution 58: 925-931.

Hall MC, Willis JH (2005). Transmission ratio distortion in intraspecific hybrids of Mimulus guttatus, implications for genomic divergence. Genetics 170: 375-386.

Hartl DL (1975). Modifier theory and meiotic drive. Theor Popul Biol 7: 168-174.

Hartl DL, Hiraizumi Y, Crow JF (1967). Evidence for sperm dysfunction as the mechanism of segregation distortion in Drosophila melanogaster. Proc Natl Acad Sci USA 58: 2240-2245.

Hiraizumi Y, Crow JF (1960). Heterozygous effects on viability, fertility, rate of development, and longevity of Drosophila chromosomes that are lethal when homozygous. Genetics 45 1071-1083

Hernández JL, Ortego J, Calabuig G, Bonal R, Muñoz A, García $G$ et al. (2007). Casi dos décadas anillando primillas en La Mancha. In: Casas F, Arredondo A, López-Jamar J (eds). Anuario Ornitológico de Ciudad Real, SEO-Ciudad Real: Ciudad Real. Vol 2003-2004, pp 165-170.

Hiraizumi Y, Dandler L, Crow JF (1960). Meiotic drive in natural populations of Drosophila melanogaster. III. Populational implications of segregation-distorter locus. Evolution 44: 433-444.

Hurst GDD, Werren JH (2001). The role of selfish genetic elements in eukaryotic evolution. Nat Rev Genet 2: 597-606.

Jaenike J (2008). X chromosome drive. Curr Biol 18: R508-R511.

Jenczewski E, Gherardi M, Bonnin I, Prosperi JM, Olivieri I, Huguet T (1997). Insight on segregation distortions in two intraspecific crosses between annual species of Medicago (Leguminosae). Theor Appl Genet 94: 682-691.
Jiggins FM, Hurst GDD, Majerus MEN (1999). How common are meiotically driving sex chromosomes? Am Nat 154: 481-483.

Kuittinen H, de Haan AA, Vogl C, Oikarinen S, Leppälä J, Koch M et al. (2004). Comparing the linkage maps of the close relatives Arabidopsis lyrata and A. thaliana. Genetics 168: 1575-1584.

LeMaire-Adkins R, Hunt PA (2000). Nonrandom segregation of the mouse univalent $X$ chromosome: evidence of spindlemediated meiotic drive. Genetics 156: 775-783.

Lewontin RC (1968). The effect of differential viability on the population dynamics of $t$-alleles in the house mouse. Evolution 22: 262-273.

Lewontin RC, Dunn LC (1960). The evolutionary dynamics of a polymorphism in the house mouse. Genetics 45: 705-722.

Liberman U (1976). Modifier theory of meiotic drive: is Mendelian segregation stable? Theor Popul Biol 10: 127-132.

Lyon MF (1984). Transmission ratio distortion in mouse $t$-haplotypes is due to multiple distorter genes acting on a responder locus. Cell 37: 621-628.

Lyttle TW (1991). Segregation distorters. Ann Rev Genet 25: 511-557.

Lyttle TW (1993). Cheaters sometimes prosper: distortion of Mendelian segregation by meiotic drive. Trends Genet 9: 205-210.

Maynard-Smith J, Haigh J (1974). The hitchhiking effect of a favourable gene. Genet Res 23: 23-35.

McDaniel SF, Willis JH, Shaw AJ (2007). A linkage map reveals a complex basis for segregation distortion in an interpopulation cross in the moss Ceratodon purpureus. Genetics 176: 2489-2500.

McMeniman CJ, Barker SC (2006). Transmission ratio distortion in the human body louse, Pediculus humanus (Insecta: Phthiraptera). Heredity 96: 63-68.

Nesje M, Roed KH, Lifjeld JT, Lindberg P, Steen OF (2000). Genetic relationships in the peregrine falcon (Falco peregrinus) analysed by microsatellite DNA markers. Mol Ecol 9: 53-60.

Ortego J, González EG, Sánchez-Barbudo I, Aparicio JM, Cordero PJ (2007). New highly polymorphic loci and crossamplified microsatellites for the lesser kestrel Falco naumanni. Ardeola 54: 101-108.

Pardo-Manuel de Villena F, de la Casa-Esperón E, Briscoe TL, Sapienza C (2000). A genetic test to determine the origin of the maternal 1: meiotic drive at the mouse Om locus. Genetics 154: 333-342.

Pardo-Manuel de Villena F, Sapienza S (2001). Nonrandom segregation during meiosis: the unfairness of females. Mamm Genome 12: 331-339.

Purushothaman D, Elliott RW, Ruvinsky A (2008). A search for transmission ratio distortions in offspring from crosses between inbred mice. J Genet 87: 127-131.

Prout T, Bundgaard J, Bryant S (1973). Population genetics of modifiers of meiotic drive. The solution of a special case and some general implications. Theor Popul Biol 4: 446-465.

Sandler L, Novitski E (1957). Meiotic drive as an evolutionary. Am Nat 91: 105-110.

Taylor DR, Ingvarsson PK (2003). Common features of segregation distortion in plants and animals. Genetica 117: 27-35.

Topinka JR, May B (2004). Development of polymorphic microsatellite loci in the Northern Goshawk (Accipiter gentilis) and cross-amplification in other raptor species. Conser Genet 5: 861-864.

Úbeda F (2006). Why Mendelian segregation? Biochem Soc Trans 34: 566-568.

Úbeda F, Haig D (2004). Sex-specific meiotic drive and selection at an imprinted locus. Genetics 167: 2083-2095.

Úbeda F, Haig D (2005). On the evolutionary stability of Mendelian segregation. Genetics 170: 1345-1357.

Weissing FJ, van Boven M (2001). Selection and segregation distortion in a sex-differentiated Population. Theor Popul Biol 60: 327-341.

Zölner S, Wen X, Hancgard NA, Herbert MA, Ober C, Pritchard JK (2004). Evidence for extensive transmission distortion in the human genome. Am J Hum Genet 74: 62-72. 